\title{
Editorial
}

\section{RIFAMPICIN/MINOCYCLINE AND OFLOXACIN (ROM) FOR SINGLE LESIONS - WHAT IS THE EVIDENCE?}

Earlier this year the results of a double-blind randomized controlled trial comparing a potential new treatment (single dose rifampicin/ofloxacin and minocycline (ROM)) for monolesion paucibacillary leprosy with the current 6-month treatment with rifampicin and dapsone (WHO-PB-MDT) were published. The executive report of the 7th WHO Expert Committee on Leprosy (Geneva, 26 May-3 June 1997) noted that the Committee considered the single-dose ROM an acceptable and cost-effective regimen for the treatment of single skin lesion PB leprosy. The paper and report have been highly influential and already strategic planners in several countries, notably India and Brazil, have introduced ROM treatment for single-lesion disease into their national programmes. We are reprinting this important paper in this issue of Leprosy Review (p. 299-300) by kind permission of the Editor of the Indian Journal of Leprosy since we feel that readers may wish to study the original publication for themselves.

The introduction of single-dose treatment for a subset of leprosy patients is obviously attractive from an operational standpoint and will make a significant impact in reducing prevalence in some areas. However it is also fraught with dangers, and hence the evidence for its effectiveness should be considered in some depth.

The trial involved nine different centres, each recruiting between 103 and 400 patients over a 10-month period to give a total of 1483 patients. Follow-up over an 18-month period was good with a $93 \%$ completion rate.

There are a number of difficulties in interpreting the data. The first of these relates to the diagnosis of leprosy in these patients. It is not clear how lesions were tested for anaesthesia nor which modalities of sensation were examined. Much of the initial testing (skin biopsy, histamine testing, lepromin testing and even detailed neurological examination) was optional. Hence it is not possible to know how many patients in the trial had definite evidence of leprosy. The system used in the Karonga trial, of grading patients on a scale of diagnostic certainty for leprosy is a useful way of addressing the problem of diagnosis. ${ }^{1}$ From an operational point of view it would be helpful to know how many patients were evaluated and prepared for entry to the trial but then proved to be slit-skin smear positive and so ineligible for treatment with ROM. There is no information on how many patients had skin biopsies with microscopic evidence of leprosy.

Children above the age of 5 were eligible for the trial but no details are given of numbers of children treated nor the drug doses used. The side-effects observed in the trial patients are briefly discussed. No mention is made of monitoring for specific side-effects and no details are given of potential side-effects such as tooth discoloration in children given minocycline. 
The major outcome measure was derived from a scoring system based on five different clinical observations of the lesions. No details of how this scale was constructed nor what weights were given to the five components nor how scoring was standardized between centres are provided. This makes interpretation of the results difficult. Of the 1381 patients who completed the trial only 12 patients failed to improve, and of these 2 deteriorated. The investigators set an improvement of 13 points in the clinical score as their definition of marked clinical improvement; their other outcome measure was complete cure. Patients treated with the conventional WHO-PB-MDT regimen showed statistically significantly better results on both these measures when compared with the patients treated with the ROM regimen. The significance level for the difference in complete cure rates is incorrectly given in Table $\mathrm{V}$ as $P=0 \cdot 04$, the correct figure is actually even more significant at $P=0.004$ as given in the text. It is not possible from the data given to discern which modalities improved most. A more detailed analysis of the data such as an analysis of covariance would have allowed examination of the effect of age, sex or type of improvement on response to treatment. This would be valuable in determining which patients would benefit most from treatment with ROM. These details are important because this trial was designed to be a gold standard trial of ROM showing its medical effectiveness, not an operational trial showing that it is an easy treatment to administer.

The follow-up period of 18 months for ROM treated patients and 12 months for WHOPB-MDT treated patients is too short to detect relapses with a relapse rate for paucibacillary disease of $1 \%$ per year. It is to be hoped that the patients will continue under active surveillance so that this important figure can be determined.

ROM is undoubtedly an attractive treatment. It is operationally easy to administer and is probably suitable for some patients. If it is to be incorporated into treatment schedules then it is important that workers follow good practice guidelines. It is vital that all patients should be examined carefully to ensure that there really is only a single lesion. Women may be reluctant to be examined fully and if so, should not be prescribed ROM. It is also vital that a careful neurological examination is done to ensure that no nerve thickening or impairment of motor or sensory function is present. There was no statistical difference in the number of reactions or neuritis in each of the treatment groups. This serves as a reminder that even patients with monolesions can suffer reactions and so need to be kept under follow-up even after single dose treatment when it will be very tempting to have less stringent follow-up.

In conclusion this trial as published leaves many doubts: how many of the patients treated in this trial actually had leprosy, which outcome measure improved, did sensation in the lesions improve, what side-effects were monitored? The analysis reveals few details but the two measures reported showed significant superiority for the existing WHO-PB-MDT regimen. Thus it is inaccurate to claim on the basis of the published data, as the authors did in their abstract that ROM is 'almost as effective as WHO-PB-MDT'. The implementation of single dose ROM should be undertaken with care; it is likely to be of value for some patients, but the attraction of operational expediency could easily result in misuse.

Editor, Leprosy Review

DIANA N. J. LOCKWOOD

Hospital For Tropical Diseases

London NW1 OPE, UK

\section{References}

${ }^{1}$ Ponnighaus JM, Fine PEM, Bliss L. Certainty levels in the diagnosis of leprosy. Int J Lep, 1986; 55: 454-462. 